\title{
In ricordo del Prof. Giuseppe Giaccone ${ }^{\dagger}$
}

\author{
Giovanni Furnari $[1]^{*}$ \\ Laboratorio di Algologia, Sezione di Biologia Vegetale del Dipartimento di Scienze Biologiche, Geologi- \\ che e Ambientali dell’Università di Catania, Italia
}

\section{Riassunto}

L'11 giugno 2018 è morto a Baucina (PA), all'età di 82 anni il Prof. Giuseppe Giaccone, già Ordinario di Botanica sistematica presso l'Università degli studi di Catania e socio emerito dell' Accademia Gioenia. Dopo gli studi liceali presso il Liceo Don Bosco di Palermo e completato nel 1960 il corso quadriennale di Teologia presso il Seminario Maggiore di Palermo, Giuseppe Giaccone si è laureato nel 1964 presso l'Università di Palermo. Nel 1973 è stato nominato assistente ordinario presso l'Università di Trieste e nel 1977 è stato trasferito all'Università di Palermo. Nel 1984 è diventato Professore associato presso l'Università di Palermo e nel 1986 Professore ordinario presso la Facoltà di Scienze Matematiche, Fisiche e Naturali dell'Università di Catania. La sua attività di ricerca ha coperto praticamente tutti gli aspetti dell'Algologia: dalla tassonomia alla fitosociologia, dall'ecologia alla valutazione di impatto ambientale e ai biodeteriogeni. I risultati delle sue ricerche sono stati divulgati attraverso conferenze, comunicazioni a convegni e congressi nazionali ed esteri e in più di 250 pubblicazioni scientifiche su riviste nazionali ed internazionali.

\section{In memory of Prof. Giuseppe Giaccone}

\section{Summary}

Prof. Giuseppe Giaccone, retired full Professor of Systematic Botany at Catania University, member emeritus of the Gioenia Academy, died on June $11^{\text {th }}$ 2018, at the age of 82. After the high school at the Liceo Don Bosco of Palermo and having completed the four-year course of theology at the Major Seminary in Palermo, Giuseppe Giaccone graduated at Palermo University in 1964. In 1973 became full assistant at the University of Trieste and in 1977 he was transferred to the University of Palermo. In 1984 he became associate Professor of Algology at the University of Palermo and in 1986 full Professor of Botany at the Faculty of Science Mathematics, Physics and Natural of the University of Catania. His research activity dealt with nearly all aspects of Algology: taxonomy, phytosociology, ecology, Environmental Impact Evaluation, biodeteriorating organisms. Results of his research were disseminated through conferences, communications at Italian and foreign meetings and congresses and were published in more than 250 papers in both national and international journals.

\footnotetext{
†Nota presentata nell'adunanza dell'8 marzo 2019

*e-mail: furnari.giovanni41@gmail.com
} 


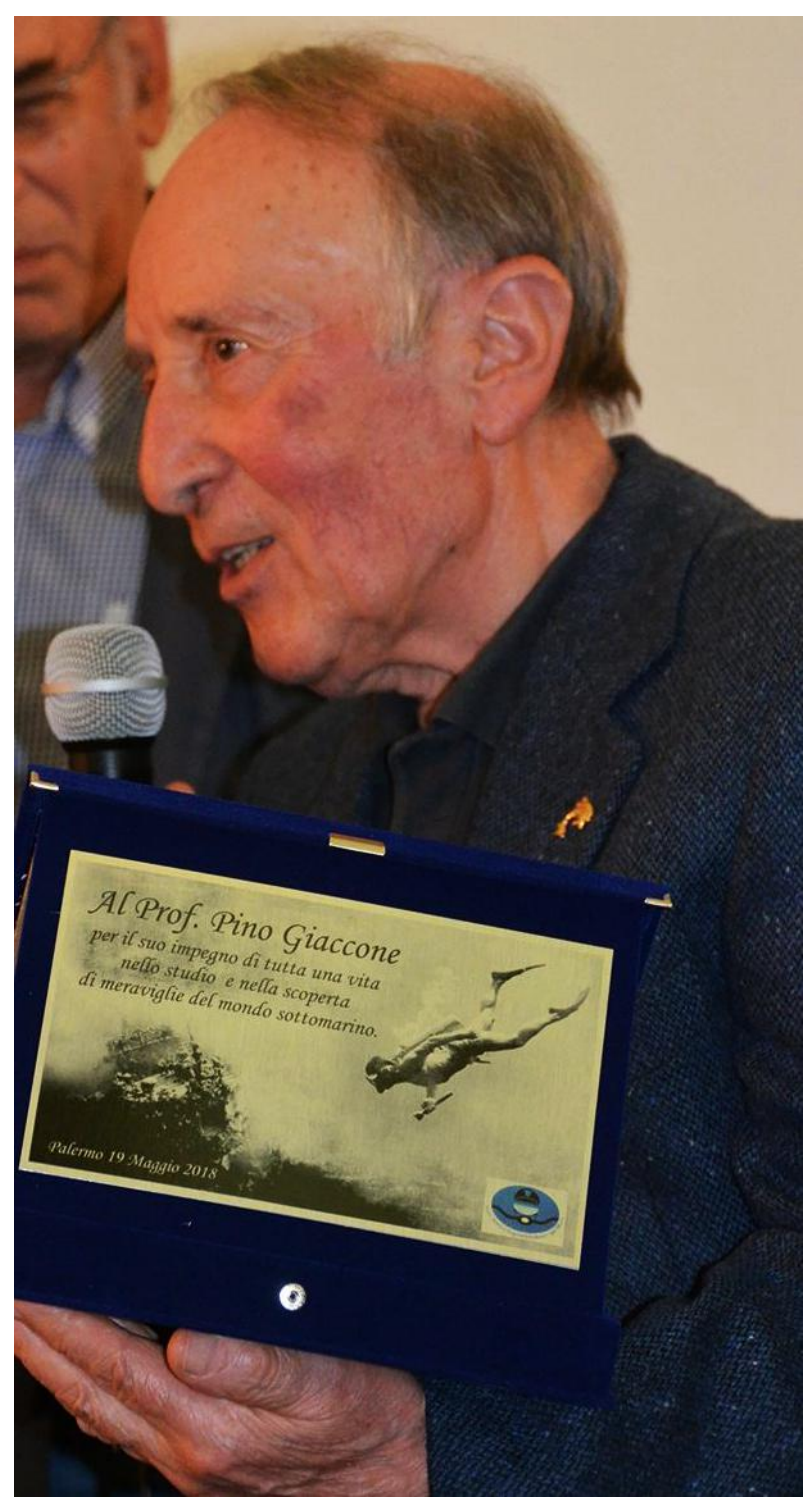

Figura 1 - prof. Giuseppe Giaccone

Giuseppe Giaccone nato a Baucina (PA) il 10.02.1936, ha conseguito la maturità classica presso il Liceo Don Bosco di Palermo ed ha completato a pieni voti nel 1960 il corso quadriennale di Teologia presso il Seminario Maggiore di Palermo. Si è laureato con lode in Scienze Naturali nel 1964 presso l'Università di Palermo, sostenendo una tesi in Algologia. Lo stesso anno è stato nominato assistente volontario e tecnico laureato di ruolo presso la cattedra di Botanica dell'Università di Palermo. Nel 1965 ha vinto una borsa di studio biennale del Ministero della Pubblica Istruzione presso la Stazione Zoologica di Napoli ove rimase fino al 1967, anno in cui fu chiamato presso l'Università di Trieste come Ricercatore del C.N.R con un contratto triennale nell'ambito del Programma finalizzato "Oceanografia e Fondi Marini”. Nel 1969 ha conseguito la Libera Docenza in Algologia e nel 1970 gli è stato conferito l'incarico esterno dell'insegnamento di Botanica sistematica presso l'Università di Ferrara. Nel settembre del 1971 ha partecipato alla prima crociera della Nave Oceanografica Bannock nel Canale di Sicilia organizzata da Paolo Colantoni dell'Istituto di Geologia Marina del C.N.R. di Bologna. Nel 1972 gli è stato conferito l'incarico dell'insegnamento di Algologia presso l'Università di Trieste. Nel 1973 viene nominato assistente ordinario presso quella stessa Università ed insegna Algologia come professore incaricato stabilizzato. Nel 1977 è trasferito come assistente ordinario all'Università di Palermo dove insegna, come professore incaricato stabilizzato, Botanica sistematica. Ha ricoperto tra il 1978 ed il 1989 le cariche di Assessore e di Sindaco presso il Comune di Baucina (PA). Nel 1984 è nominato Professore Associato di Algologia nell'Università di Palermo. Nel 1986 è nominato Professore Ordinario di 
Botanica presso l'Università di Catania, dove svolgerà la sua attività didattica e di ricerca sino al suo pensionamento avvenuto nel novembre del 2008. Presso l'Università di Catania ha ricoperto gli insegnamenti di Algologia presso il Corso di Laurea in Scienze Naturali, di Ecologia generale presso il Corso di Laurea in Scienze ecologiche ed educazione ambientale, di Biologia delle Alghe, di Progettazione di VIA e di VAS, di Storia della Scienza presso il Corso di Laurea Specialistica in Scienze ecologiche. Dal 2003 al 2005 ha anche insegnato la disciplina Biodeteriogeni dei Beni Culturali presso il Corso di Laurea in Conservazione e Restauro dei Beni Culturali dell'Università di Palermo. Ha inoltre tenuto lezioni teorico-pratiche di formazione per tecnici di controllo ambientale, per operatori ecologici, per esperti in Valutazione di Impatto Ambientale e per esperti in aree protette e parchi naturali, in corsi tenuti sotto la sorveglianza dell'Assessorato Regionale al Lavoro. Ha tenuto altri corsi per conto del Politecnico di Milano e dell'U.N.E.S.C.O. presso le Università di Lubiana (Slovenia) e di Granada (Spagna). Ha svolto attività di tutor e di esaminatore esterno per tesi e commissioni di esami di Master e di PHD presso le Università di Catania, di Lubiana (Slovenia), di Malta e di Algeri. Nel 2002 ha ricoperto il ruolo di docente nella Scuola superiore di Catania per la formazione di eccellenza per il Master in Valutazione di Impatto Ambientale e per il Master in Economa dei Beni Culturali. Negli anni acc. dal 2001/2002 al 2005/2006 è stato Presidente del Corso di Laurea triennale in Scienze ecologiche ed Educazione ambientale della Facoltà di Scienze Matematiche, Fisiche e Naturali dell'Università di Catania e nel 2006/2007 è stato nominato Presidente della "Struttura Didattica Aggregata in Scienze ecologiche" risultante dall'aggregazione del suddetto Corso di laurea triennale con il nuovo Corso di Laurea specialistica in Scienze Ecologiche.

Ho incontrato "Pino" Giaccone nel 1969 nel corso di una crociera di studio alle Isole Eolie effettuata su un peschereccio trasformato in nave da ricerca che si chiamava Entella. Si trattava della mia prima esperienza di apprendista algologo. Lì, insieme all' amico Blasco Scammacca, ho ascoltato proprio da Pino le prime lezioni di Algologia. Fu così che sia io che Blasco Scammacca decidemmo di continuare a studiare le alghe, utilizzando per la determinazione delle specie, proprio il suo lavoro "Elementi di Botanica Marina" (una pubblicazione della serie didattica dell'Istituto Botanico dell'Università di Trieste): le mitiche "Chiavi di Giaccone", ampiamente utilizzate dagli algologi italiani in quegl'anni. Nei mesi di maggio e ottobre 1973 partecipai insieme a Blasco Scammacca, Francesco Cinelli, Mario Sortino, Attilio Solazzi, Claudio Tolomio e naturalmente Pino Giaccone alle mie prime campagne di studio algologico all'isola di Linosa. Lì ebbi modo di conoscere meglio Pino, con il quale si creò una reciproca amicizia. Da allora nacque anche una collaborazione scientifica che si concretizzò in numerose pubblicazioni. I rapporti, naturalmente si fecero più intensi dal 1986, anno in cui Pino rivestì il ruolo di Professore Ordinario nel settore scientifico disciplinare "Botanica sistematica" presso l'Università di Catania e ancor di più dal 2001, quando mi nominò "fedele" Vice Presidente delle strutture didattiche da lui presiedute e mi incaricò di fare da Segretario in tutte le sedute dei relativi Consigli.

Il Prof. Giuseppe Giaccone è stato socio effettivo dell'Accademia Gioenia, nella Sezione di Scienze della Natura, a partire dal 21.11.1995 ed è passato nella categoria "emerito" nel febbraio 2011. Nel triennio 1999-2001 ha svolto le funzioni di Segretario generale (Presidente: Prof. Attilio Agodi). Ha fatto inoltre parte del Consiglio di Presidenza, quale componente, nel sessennio 2002-2007, sotto la Presidenza del Prof. Salvatore Foti e poi dall'1.01.2011 al 26.10.2011, sotto la Presidenza del Prof. Angelo Messina. Giuseppe Giaccone è stato insignito dall'Accademia dei Lincei del "Premio Grassi" per la Talassografia biologica. Ha ricevuto il premio nazionale "Maurizio Sarra" per la fotografia biologica subacquea. È stato insignito del premio "Tridente d'Oro" per le Attività scientifiche ed iperbariche dall'Accademia Internazionale di Scienze e Tecniche Subacquee. È stato insignito del premio "Castore e Polluce" dalla Lega Navale Italiana come "Uomo del Mare 1990". È stato socio di varie associazioni ambientaliste e scientifiche (S.B.I., S.I.B.M., Società dei Naturalisti Siciliani, Ente Fauna Siciliana, Marevivo) anche con compiti negli organi direttivi. È stato Vice-presidente dell'Accademia del Mare (ONLUS), socio corrispondente dell'Académie Europeénne, volontario nel Movimento dei Focolari fondato da Chiara Lubich, componente esterno della Scuola Abba nel Gruppo Scienze della Natura e della Commisione in- 
ternazionale "EcoOne", Ecologia per un mondo unito, di questo Movimento ecclesiale. Ha fatto parte del Consiglio Direttivo del CoNISMA e dell'Istituto Italo-Russo di Formazione e Ricerche ecologiche come delegato del Rettore dell'Università di Catania. È stato componente nell'Università di Catania del Centro di Ricerche sulle Cause di Degrado per il Recupero dei Beni Culturali e Monumentali. È stato nominato dal Vescovo di Caltagirone membro del Consiglio Scientifico del Museo S. Nicolò di Militello (CT). Ha svolto attività di consulenza per le Nazioni Unite e per le agenzie U.N.E.P., RAC/SPA. In particolare, ha redatto il documento preparatorio per il Protocollo di Atene della Convenzione di Barcellona sulle fonti terrestri dell'inquinamento, insieme ad altri 30 esperti internazionali, nonché alcuni Piani di Azione del P.A.M., tra i quali nel 1999 il Piano di Azione per la Protezione della Vegetazione marina del Mediterraneo.

Inoltre ha collaborato nella redazione della lista dei Tipi di Habitat marini per il Mediterraneo, dei Criteri (Standard Data Form) per la caratterizzazione delle Aree Specialmente Protette di Interesse per il Mediterraneo (ASPIM) e ha redatto una memoria sullo stato delle conoscenze della tassonomia dei vegetali marini del Mediterraneo, per l'Iniziativa Mediterranea per la Tassonomia (IMT), nell'ambito della Convenzione Mondiale per la Biodiversità. Nel 2005 è stato incaricato dall'UNEP/PAM/RAC/SPA di dirigere una sessione di formazione per ricercatori dei paesi del sud e dell'est del Mediterraneo per attuare in essi l'IMT, promuovendo le collezione di riferimento dei vegetali marini. È stato convocato presso la Commissione Ecologia del Senato nel corso di audizioni di esperti in preparazione della Legge 319/76 sull'inquinamento idrico. Ha fatto parte di Commissioni regionali sull'ambiente e l'energia della Sicilia, delle Regioni Friuli-Venezia Giulia ed Emilia-Romagna. Ha compilato per conto del Ministero dell'Ambiente l'elenco annotato dei vegetali marini delle coste italiane, le carte di distribuzione, le schede descrittive per la conservazione degli Habitat prioritari lungo i mari dell'Italia finalizzate a documentare la Biodiversità dell'Italia, nell'ambito delle direttive CE e degli accordi UNEP. Su invito dell'Accademia di Francia ha tenuto una relazione al Seminario sulle alghe verdi del genere Caulerpa aliene ed invasive nel Mediterraneo. Ha compiuto studi ambientali pluridisciplinari per incarico della Regione Toscana, delle Province di Crotone, di Palermo e di Ragusa. Ha coordinato nel 1990-1991 la Valutazione di Impatto Ambientale per gli scarichi delle città di Palermo e di Catania per incarico dell' ITALISPACA su concessione della Presidenza del Consiglio dei Ministri. Ha svolto studi ambientali per conto di imprese a partecipazione statale (Enichem, Montedison, Eurallumina, Anic, Agip) a Cagliari, Portovesme, Ravenna, dell'ARPA in Sicilia (AMP del Plemmirio presso Siracusa) ed in Calabria (Gioia Tauro) per conto del Tribunale. Ha avuto la supervisione (quadro di riferimento ambientale: ambiente idrico) della V.I.A. nella Baia di Santa Panagia (SR) del complesso ISAB S.p.A. di Gasificazione e Cogenerazione.

Nella sua attività di ricerca ha compiuto oltre cinquemila immersioni subacquee con A.R.A. (autorespiratore ad aria), con miscele e con sommergibili fino a 230 metri di profondità in Mediterraneo, in Atlantico e nell' Oceano Indiano in Australia. Per conto della C.I.E.S.M., ha preparato nel 1992 alcuni modelli sperimentali di Valutazione di Impatto Ambientale insieme ad esperti di varie nazioni europee. Ha prodotto monografie sulla legislazione ambientale, svolto ricerche sugli indici di stato ecologico delle acque, sul controllo degli impianti di depurazione e sul controllo dell'inquinamento da fitofarmaci per conto dell' Assessorato Regionale alla Sanità e del Ministero per l'Università e la Ricerca scientifica e tecnologica. Ha partecipato alla redazione del piano dei vincoli e del regolamento di gestione per le Riserve Nazionali Marine di Miramare, Ustica, Isole Ciclopi, Isole Egadi e allo studio di fattibilità per le Isole Pelagie, per le Isole Eolie e ha diretto lo studio di fattibilità dell' AMP della Penisola Maddalena e di Capo Murro di Porco (oggi del Plemmirio). Ha svolto studi sulla vegetazione sommersa di varie riserve marine e costiere (Miramare, Ustica, Vendicari, Timpa di Acireale, Isola di Capo Rizzuto, Capo Gallo e Isola delle Femmine, Pantelleria, Monte Cofano, Capo Passero e Isola delle Correnti) e ha fatto parte della Commissione di Riserva per le Isole Ciclopi su nomina del Ministero dell' Ambiente. 


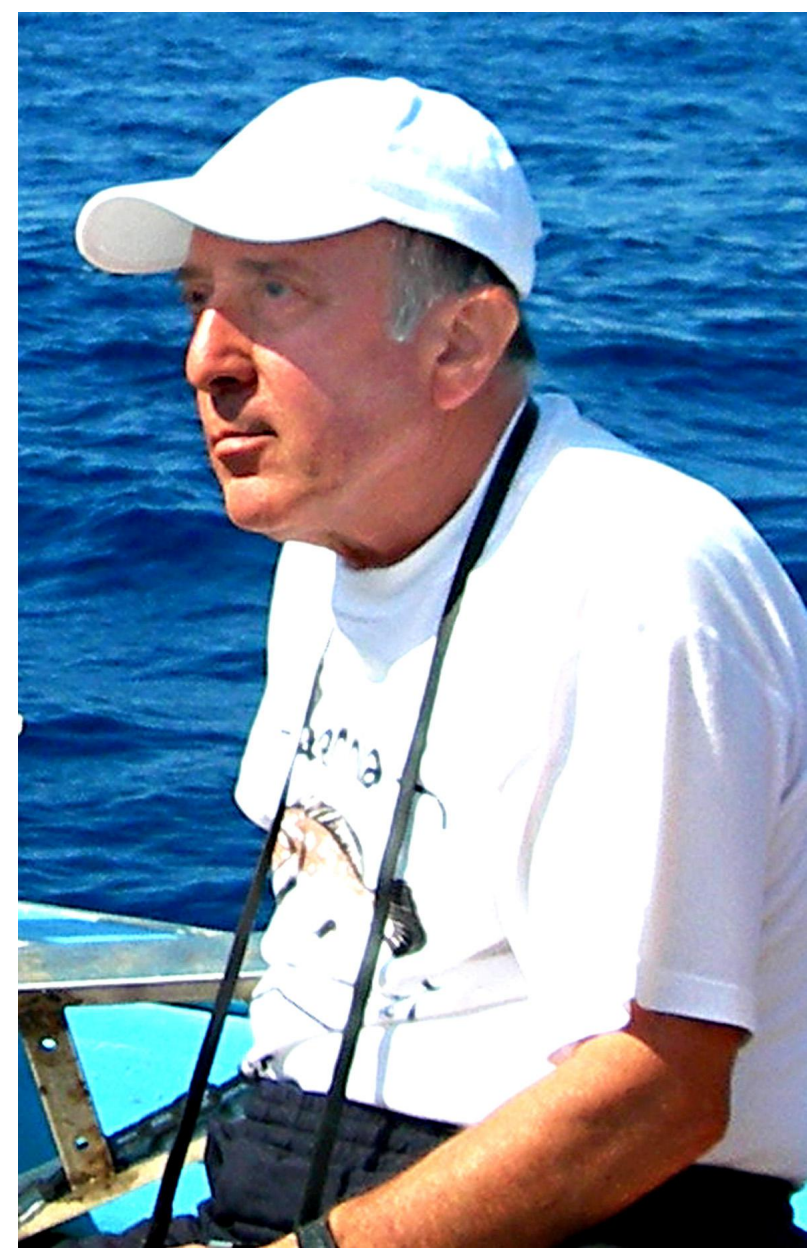

Figura 2 - prof. Giuseppe Giaccone

L'attività di ricerca di Giuseppe Giaccone si può dire che abbia interessato tutti i campi dell'algologia. La ricerca di base ha riguardato la tassonomia delle alghe, la vegetazione marina, salmastra ed alofila. La sua ricerca applicata è stata rivolta ad argomenti di legislazione e di politica ambientale, di gestione delle risorse per lo sviluppo sostenibile; a tematiche concernenti le aree protette, l'acquacoltura, l'inquinamento delle acque marine, l'impatto industriale sui popolamenti marini, le specie marine aliene introdotte o migrate, la depurazione e lo smaltimento dei reflui urbani ed industriali; a progetti esecutivi di ripopolamento mediante barriere artificiali e riforestazione sottomarina, a progetti riguardanti i biodeteriogeni delle opere d'arte, per i quali ha collaborato con ricercatori dell'Istituto Centrale del Restauro di Roma. Oltre ai suoi studi algologici, Giuseppe Giaccone ci ha pure lasciato delle profonde riflessioni su temi spirituali e religiosi con i quali si era confrontato in età giovanile e che non lo avevano mai lasciato nel suo percorso personale e professionale. Basti ricordare quelle sulla visione spirituale del rapporto tra l'uomo e la natura, sull'etica ambientale, sulle radici religiose dell' ambientalismo nelle religioni monoteiste (Ebraismo, Cristianesimo, Islam) che riconoscono Abramo come comune padre nella fede in un unico Dio.

Ma, se posso esprimere un parere personale, le sue più grandi passioni scientifiche sono state lo studio della vegetazione marina e delle Cistoseire (Alghe brune). Lo studio della vegetazione marina del Mediterraneo e del suo inquadramento fitosociologico si è concretizzato nella pubblicazione di fondamentali monografie riguardanti i taxa fitosociologici (molti dei quali descritti e/o emendati dallo stesso Giaccone) presenti in tutti gli ambienti del Mediterraneo: dal sopralitorale al circalitorale, dagli ambienti in equlibrio a quelli più o meno alterati fino a quelli interessati dalla presenza di specie aliene. Un discorso a parte merita la sua ricerca sulle Cistoseire che, come ho definito in una canzone a lui dedicata, erano per lui come "figlie" che lui da buon "papà" conosceva alla perfezione. Il binomio Giaccone-Cistoseire era e rimane un "assioma". Egli infatti ha 
studiato le Cistoseire dal punto di vista tassonomico (con metodo classico, numerico e chemotassonomico), biologico ed ecologico. Pertanto, per qualsiasi problema riguardante quel genere, era lui il punto di riferimento. I risultati delle sue ricerche sono stati divulgati attraverso conferenze, comunicazioni a convegni e congressi nazionali ed esteri e in più di 250 pubblicazioni scientifiche su riviste nazionali ed internazionali. Giuseppe Giaccone è morto a Baucina (PA) l'11/06/2018. Con lui l'Algologia ha perso un Maestro ed io ho perso un amico. 